\title{
A case with myasthenia gravis, brain stem multiple infarcts, fracture of vertebrae Th6 and discal hernia to the Th7/Th8
}

\author{
Vera Kukaj ${ }^{1}$, Shpresa Beqiri ${ }^{1}$, Melihate Pushka ${ }^{1}$, \\ Myrvete Kabashi ${ }^{1}$, Edmond Komoni ${ }^{1}$ \\ ${ }^{1}$ Department of Neurology, University Clinical Center of Kosovo, Pristine, Republic of Kosovo
}

\begin{abstract}
Background. We report the case of a patient with myasthenia gravis accompanied with brain stem multiple infarcts, fracture of vertebrae Th6 and discal hernia of the Th7/TH8.

Case report. A 66-year-old male patient, one week prior to the hospitalization showed up complaints of dizziness, nausea, vomiting, numbness of the left side of the face, swallowing difficulty, left side body weakness, right side of the body numbness starting from the nipples and going down to the right leg as well as general fatigue. Six years ago the patient was diagnosed with myasthenia gravis based on electrophysiological investigations, pharmacologic tests and findings of acetylcholine (Ach) receptor antibodies in serum. He was then treated with the following medications: pyridostigmine of 60mg $x$ 5/day, prednisolon of $20 \mathrm{mg}$ (every other day), azathioprine 100mg tid. He was doing well under described therapy. Twenty years ago his left kidney was removed due to calculosis. He had also a sister that suffered of myasthenia gravis and diabetes mellitus.
\end{abstract}

Conclusions. Myasthenia gravis has a number of symptoms and signs which probaly are in common with stroke-including fatigue, muscle weakness, slurred speach and swallowing difficulty. The reported case supports the opinion that several medical conditions such as brain steam stroke may mimick myasthenia gravis.

Key words: myasthenia gravis; brain stem multiple infarcts; fracture of vertebrae

\section{Introduction}

Myasthenia gravis (MG) is an autoimmune disorder of peripheral nerves in which antibodies are formed against acetylcholine

Received 18 November 2008

Accepted 23 January 2009

Correspondence to: Vera Kukaj, MD, PhD, Department of Neurology, University Clinical Center of Kosovo, Pristine, Kosovo. Phone: + 37744185 620; Email: verekukaj@hotmail.com nicotinic postsynaptic receptors (AChRs) at the myoneuronal junction. A reduction in the number of AChRs, results in a characteristic pattern of progressively reduction of muscle strength with repeated use of the muscle and recovery of muscle strength following a period of rest. ${ }^{1-4}$ The bulbar muscles are affected most commonly and most severely, but most patients also develop some degree of intermittent generalized weakness. ${ }^{3}$ Auto antibodies develop against $\mathrm{AChRs}$ nicotinic postsynaptic 
receptors for unknown reasons, although certain genotypes are more susceptible. The presynaptic terminal contains vesicles filled with acetylcholine (ACh). On arrival of a nerve action potential, the contents of these vesicles are released into the synaptic cleft in a calcium-dependent manner. The released $\mathrm{ACh}$ molecules diffuse across the synapse and bind to the AChRs on the postsynaptic membrane. . $^{3,4}$

Anti-AChR antibody is found in approximately $80-90 \%$ of patients with MG. Patients with anti-AChR antibodies are recognized as seropositive myasthenia gravis (SPMG). Patients become symptomatic once the number of AChRs is reduced to approximately $30 \%$ of normal. Patients without antiAChR antibodies are recognized as seronegative myasthenia gravis (SNMG). Many of these patients with SNMG have antibodies against muscle-specific kinase (MuSK). MuSK plays a critical role in postsynaptic differentiation and clustering of AChRs., ${ }^{2,4}$

Immunogenic mechanisms play important roles in the pathophysiology of myasthenia gravis. Supporting clinical observations include the presence of associated autoimmune disorders in patients suffering from myasthenia gravis (e.g. autoimmune thyroiditis, systemic lupus erythematosus, rheumatoid arthritis). The role of the thymus in the pathogenesis of myasthenia gravis is not entirely clear, but $75 \%$ of patients with myasthenia gravis have some degree of thymus abnormality (e.g. hyperplasia in $85 \%$ of cases, thymoma in $15 \%$ of cases). Given the immunologic function of the thymus and the improvement in the clinical condition of patients following thymectomy, the thymus is suspected to be the site of autoantibody generation. However, the stimulus that initiates the autoimmune process has not been identified.

The most common symptoms are: dropping of the eye lids, double vision, slurred speech, swallowing and chewing difficulty, muscle weakness of arms and legs, chronic fatigue and sometimes breath difficulty. ${ }^{1-6}$

To facilitate the clinical staging of therapy and prognosis, the following classification, introduced by Osserman, has been widely adopted: ${ }^{1}$

I Ocular myasthenia (15-20\%).

IIA Mild generalized myasthenia with slow progression, no crises: drug responsive $(30 \%)$.

IIB Moderately severe generalized myasthenia; severe skeletal and bulbar involvement but no crisis, drug response less than satisfactory (25\%).

III Acute fulminating myasthenia; rapid progression of severe symptoms with respiratory crises and poor drug response; high incidence of thymoma; high mortality (15\%).

IV Late severe myasthenia; symptoms the same as III, but resulting from steady progression over 2 years from class I to class II (10\%).

Female to male ratio in children and adults is $3: 2$. It can manifest at any age from the birth until the 80 -ties. The peak age of onset is between 20 and 30 years in women and between $50-60$ years in men. Under the age of 40 , females are affected two to three times as often as males, whereas in later life, the incidence in males is higher $3: 2{ }^{1-4}$ The diagnosis is made by the following; pharmacologic investigations: edrophonium chloride (Tensilion) test, pyridostigmine (Prostigmin) test. electrophysiological investigations; repetitive nerve stimulation test, single fiber EMG studies (SFEMG) and serologic tests; serum AChR antibody test, muscular specific thyrosin kynase (antiMuSk) antibodies. ${ }^{4-8}$ The treatment consists of cholinesterase inhibitors, immunosuppressant, thymectomy, plasmapheresis and i.v. immunoglobulins. ${ }^{9-13}$ 


\section{Case report}

The patient was a 66-year old male. Six days prior to the hospitalization the patient showed up with complaints of dizziness, nausea, vomiting, numbness of the left side of the face and body, difficulty in swallowing, left side body weakness, right side of the body numbness starting from the nipples and down the right leg and general fatigue. Also six years ago the patient was diagnosed with myasthenia gravis (generalized form, Osserman II.B) with electrophysiological investigations, pharmacologic tests and findings of acetylcholine receptor antibodies in serum. For years his conditions were good with the following treatment: pyridostigmine (Mestinon) tablets of $60 \mathrm{mg} 5 \times 1$, prednisolon tablets of 20 mg 1x1 (every second day), azathioprine (Imuran) tablets of $100 \mathrm{mg} 2 \times 1$. He was doing well with the described therapy. Twenty years ago his left kidney was removed due to calculosis and he suffered from hypertension, too, which he treated with lisinopril and hydrochlorothiazide tablets of 10 mg $1 \times 1$. He had a sister also diagnosed with myasthenia gravis and diabetes.

\section{Neurologic examination}

He suffered from horizontal nystagmus on the right, diplopia, dysphagia, dysphonia, supranuclear paresis of the left facial nerve, the uvula deviated on the right, hemihypaesthesia on the left side of face, muscular weakness of jaw muscles and hemihypaesthesia on the left.

Upper extremities: muscle strength was weaker on the left side (grade 4), there was reflex asymetry presented (again weaker on the left). We found hypaesthesia for all qualities on the right side with the sensory level to the Th 5/6 vertebrae.

Lower extremities: muscular tone was weaker on the left side, muscle strength

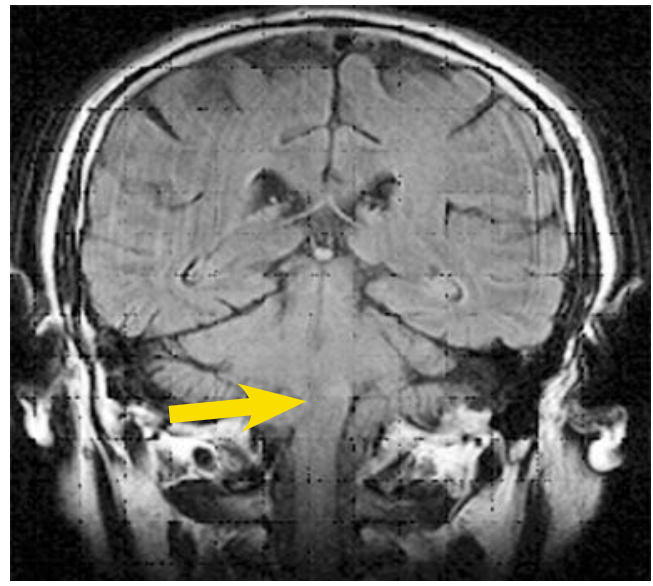

Figure 1. Brain MRI coronal FLAIR shows ischemic lesions in the brain stem (On the caudal part of the left side of brain stem, there is a hiperintense area with $5 \times 6 \mathrm{~mm}$ dimensions and discrete hiperintense areas are present on the both sides of the pons).

was also weaker on the left, there was hiperreflexia bill.

Superficial abdominal reflexes were weaker on the left. Extensor plantar reflex (Babinski) was present left. Rombergs test was positiv. Gait was ataxic. Primitive reflexes: it was snout response presented.

\section{Other investigations}

On investigation the full blood count and routine biochemistry were normal. Screening with echocardiogram and X-ray chest were also normal. Without changes were TSH, T3 and T4 levels. Brucella tests and rheumatologic tests were negative. Prostate specific antigen (PSA) was negative. The abdominal ultrasound examination: the left kidney was missing (extirpated), the right one was with compensatory hypertrophy but without any urine present, ultrasound of carotid of blood vessels did no reveal stenosis or occlusion, CT of brain was normal. Brain MRI showed ischemic lesions in the brain stem (Figure 1). MRI of the spine cervical thoracic level revealed a fracture of the Th6 vertebrae, disc hernia to 
the Th7/8 with narrowing the spinal canal and signs of spinal compression in this level with multiple protrusions of intervertebral discs in cervical and thoracic spine (Figure 2).

A cardiologic consultation established Hypertensio arterialis and physiatrist sugested physical therapy.

\section{Discusion}

Myasthenia gravis is one of the most challenging diseases when it comes to diagnosis and treatment. ${ }^{1,3}$ Little is known about ishemic stroke in patients with myasthenia gravis. It is well known that several medical conditions may mimick stroke; however, myasthenia gravis mimicking stroke apears to be uncommon. ${ }^{14,15}$ That is comprehensive, since myasthenia gravis has a number of symptoms in common with stroke-including fatigue, muscle weakness, slurred speach and swallowing difficulty. We presented here a patient with myasthenia gravis and brain stem stroke suffering a lot of symptoms as exacerbation of myasthenia gravis. Our patient with mysthenia gravis, developed ischemic lesions in the brain stem and also degenerative changes in the spinal cord with intervertebral right disc hernia to the Th7/8 and disc protrusions to the Th2/3 and Th6/7 level together with the fracture of Th6 vertebre. The vertebral and spinal cord patology was cleared up with MRI, which is still the best diagnostic modality providing to the clinician the best information. ${ }^{16}$ We don't have data regarding eventual trauma. He has several risc factors for ischemic brain disease: hypertension, and he has been receving for six years now corticosteroids, immunossupresants and anticholinergic drugs. ${ }^{15}$ Age of the patient, risc factors for cerebrovascular disease and the history of treatment for six years with corticosteroids and immunossupresive dru-

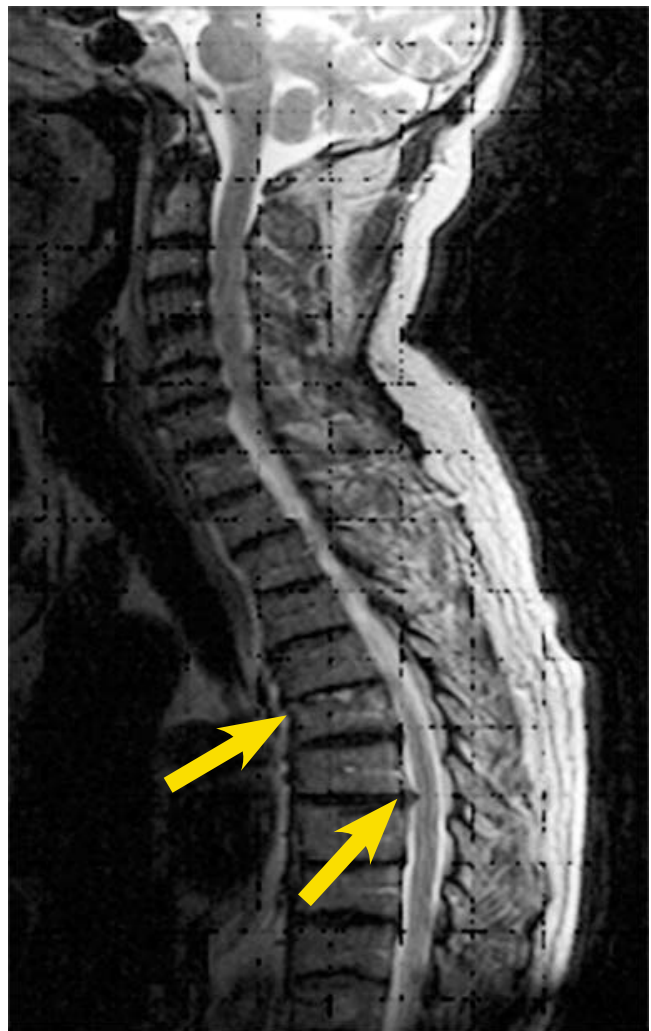

Figure 2. T2 MRI (sagittal image) of the spine cervicalthoracic level shows the fracture on the upper margin of the Th6. The intervertebral disc C3/C4 is presented with a dorsal median expansion. The intervertebral discs to the $\mathrm{C} 5 / \mathrm{C} 6$ and $\mathrm{C} 6 / \mathrm{C} 7$ are presented with a dorsal median expansion. The intervertebral discs to the Th2/Th3 and Th6/Th7 are presented with circular protrusions. Disc hernia to the Th7/Th8 with right dorsal paramedian expansion and narrowing of the spinal canal and signs of spinal compression.

gs indicate the possible linkage between ischemic changes in the brain stem and degenerative changes in the spinal colum with the therapy mentioned above for a long period of time. ${ }^{12,15}$

\section{Conclusions}

Myasthenia gravis has a number of symptoms and signs which may be in common with stroke-including fatigue, muscle 
weakness, slurred speach and swallowing difficulty. The reported case supports the opinion that several medical conditions as brain steam stroke may mimick myasthenia gravis, too.

The reason for presenting this case is to emphasize similarities and differences between these two disorders, especially when it comes to life threatening situations to which both can lead the particularly regarding treatment which usually is very different in different diseases. As for the described findings in the spinal cord without evidence of trauma in the past, we think that it is a case of casually comorbidity.

\section{References}

1. Victor M, Ropper A. Myasthenia gravis and related disorders of the neuromuscular junction. In: Adams and Victors principles of neurology. Victor $\mathrm{M}$, Ropper A, Adams RD, editors. VIIth ed. New York: McGraw-Hill; 2001. p. 1536-51.

2. Juel VC. The clinical feature and diagnosis of myasthenia gravis, Update on myasthenia gravis. AAEM 51 $1^{\text {st }}$ Annual Scientific Meeting, Savannah, Georgia 2004. p. 7-12.

3. Newton E. Myasthenia gravis. e-medicine 2007.

4. Shah A. Myasthenia gravis. e-medicine 2006.

5. Keesey JC. Clinical evaluation and management of myasthenia gravis. Muscle Nerve 2004; 29: 484-505.

6. Juel VC, Massey JM. Myasthenia gravis. Orphanet J Rare Dis 2007; 2: 44.

7. Donald B Sanders. The MuSK antibody-positive myasthenia gravis. Update on myasthenia gravis 2004. AAEM 51 ${ }^{\text {st }}$ Annual Scientific Meeting, Savannah, Georgia, 2004. p.13-8.

8. Agius MA, Richman DP, Fairclough RH, Aarli J, Gilhus NE, Romi F. Three forms of immune myasthenia, Ann N Y Acad Sci 2003; 998: 453-6.

9. Vincent A, Palace J, Hilton-Jones D. Myasthenia gravis. Lancet 2001; 357: 2122-8.

10. Saperstein DS; Barohn RJ. Managmement of myasthenia gravis. Semin Nurolo 2004; 24: 41-8.
11. Delacas MC. Intravenous immunoglobulin in autoimmune neuromuscular disorder. JAMA 2004; 291: $2367-75$.

12. Zinman L, Bril V. IVIG treatment for myasthenia gravis: effectiveness, limitations and novel therapeutic strategies. Ann N Y Acad Sci 2008; 1132: 264-83.

13. Sivakumar Sathasivam. Steroids and immunosuppressant drugs in myasthenia gravis. Nat Clin Pract Neurol 2008; 4: 317-27.

14. Richard L, Richard B, Keneth E. Myasthenia mimicking vertebrobasilar stroke. J Neurol 2002; 249: 1512-14.

15. Vincent A, Clover L, Buckley C, Grimley Evans J, Rothwell PM; UK Myasthenia Gravis Survey. Evidence of underdiagnosis of myasthenia gravis in older people. J Neurol Neurosurg Psychiatry 2003; 74: $1105-8$.

16. Rajer M, Kovač V. Malignant spinal cord compression. Radiol Oncol 2008; 42: 23-31. 